\title{
Socially Aware Navigation: A Non-linear Multi-objective Optimization Approach
}

\author{
SANTOSH BALAJEE BANISETTY, Dept. of Computer Science and Engineering, University of Nevada \\ Reno \\ SCOTT FORER and LOGAN YLINIEMI, Dept. of Mechanical Engineering, University of Nevada \\ Reno
}

MONICA NICOLESCU and DAVID FEIL-SEIFER, Dept. of Computer Science and Engineering, University of Nevada Reno

\begin{abstract}
Mobile robots are increasingly populating homes, hospitals, shopping malls, factory floors, and other human environments. Human society has social norms that people mutually accept; obeying these norms is an essential signal that someone is participating socially with respect to the rest of the population. For robots to be socially compatible with humans, it is crucial for robots to obey these social norms. In prior work, we demonstrated a Socially-Aware Navigation (SAN) planner, based on Pareto Concavity Elimination Transformation (PaCcET), in a hallway scenario, optimizing two objectives so the robot does not invade the personal space of people. This article extends our PaCcET-based SAN planner to multiple scenarios with more than two objectives. We modified the Robot Operating System's (ROS) navigation stack to include PaCcET in the local planning task. We show that our approach can accommodate multiple Human-Robot Interaction (HRI) scenarios. Using the proposed approach, we achieved successful HRI in multiple scenarios such as hallway interactions, an art gallery, waiting in a queue, and interacting with a group. We implemented our method on a simulated PR2 robot in a 2D simulator (Stage) and a pioneer-3DX mobile robot in the real-world to validate all the scenarios. A comprehensive set of experiments shows that our approach can handle multiple interaction scenarios on both holonomic and non-holonomic robots; hence, it can be a viable option for a Unified Socially-Aware Navigation (USAN).
\end{abstract}

CCS Concepts: $\bullet$ Mathematics of computing $\rightarrow$ Mathematical optimization; $\bullet$ Human-centered computing • Computer systems organization $\rightarrow$ Robotics;

Additional Key Words and Phrases: Human-robot interaction, socially-assistive robotics, socially-aware navigation

L. Yliniemi is currently with Amazon Robotics. This work was completed prior to joining Amazon.

The authors would like to acknowledge the financial support of this work by the National Science Foundation (NSF, \#IIS1719027), Nevada NASA EPSCoR (\#NNX15AI02H), and the Office of Naval Research (ONR, \#N00014-16-1-2312, \#N0001414-1-0776).

Authors' addresses: S. B. Banisetty, M. Nicolescu, and D. Feil-Seifer, Dept. of Computer Science and Engineering, University of Nevada Reno, 1664 N. Virginia Street, Reno, NV, 89557; emails: santoshbanisetty@nevada.unr.edu, monica@unr.edu, dave@cse.unr.edu; S. Forer and L. Yliniemi, Dept. of Mechanical Engineering, University of Nevada Reno, 1664 N. Virginia Street, Reno, NV, 89557; emails: sforer@nevada.unr.edu, logan@unr.edu.

The reviewing of this article was managed by associate editor Joseph LaViola.

Permission to make digital or hard copies of all or part of this work for personal or classroom use is granted without fee provided that copies are not made or distributed for profit or commercial advantage and that copies bear this notice and the full citation on the first page. Copyrights for components of this work owned by others than the author(s) must be honored. Abstracting with credit is permitted. To copy otherwise, or republish, to post on servers or to redistribute to lists, requires prior specific permission and/or a fee. Request permissions from permissions@acm.org.

(c) 2021 Copyright held by the owner/author(s). Publication rights licensed to ACM.

2160-6455/2021/06-ART15 \$15.00

https://doi.org/10.1145/3453445

ACM Transactions on Interactive Intelligent Systems, Vol. 11, No. 2, Article 15. Publication date: June 2021. 
ACM Reference format:

Santosh Balajee Banisetty, Scott Forer, Logan Yliniemi, Monica Nicolescu, and David Feil-Seifer. 2021. Socially Aware Navigation: A Non-linear Multi-objective Optimization Approach. ACM Trans. Interact. Intell. Syst. 11, 2, Article 15 (June 2021), 26 pages.

https://doi.org/10.1145/3453445

\section{INTRODUCTION}

Social norms such as driving on the right or left side of the road (depending on the country one lives in), turn-taking rules at four-way stops and roundabouts, holding doors for people behind us, and maintaining an appropriate distance when interacting with another person (actual distance depending on the type of interaction) are crucial in our day-to-day interactions. People use these actions as signals that they are participants in the social order. Violating these principles is jarring at best (i.e., a person becoming confused at another person's behavior) and can provoke hostility at worst (i.e., getting upset at someone for cutting in line).

As socially assistive robots (SAR) [21] are expected to play an essential role in a human-robot collaborative environment, these robots taking roles while remaining unconstrained to such social norms is a growing concern in the robotics community. In the past decade, social and personal robots have attained immense interest among researchers and entrepreneurs alike; the result is an increase in the efforts both in industry and academia to develop applications and businesses in the personal robotics space. Smart Luggage [18], showcased at 2018's consumer electronics show (CES), a robotic suitcase that will follow the owner during travel, demonstrates the commercial viability of interpersonal navigation. Some companies and start-ups develop robotic assistants for airports and shopping malls to assist people with directions and shopping experiences. Robot domains, especially SAR, benefit from navigation, as such movement extends the robot's reachable service area. However, navigation, if not appropriately performed, can cause an adverse social reaction [46]. In an ethnographic study where nurses in hospital settings interacted with an autonomous service robot, one of the participants quoted the following statement:

"Well; it almost ran me over... I wasn't scared... I was just mad... I've already been clipped by it. It does hurt."

Robots currently deployed in human environments have prompted adverse reactions from people encountering them [13]. Some people are unwilling to interact with these robots, and some even kicked them, demonstrating a very hostile attitude towards service robots. Incidents such as these pose both challenges and opportunities for the human-robot interaction (HRI) researchers; as a result, recent years have seen a tremendous growth of publication in areas related to socially aware navigation such as human detection and tracking, social planner incorporating social norms, and human-robot interaction studies to understand the problem from a human's perspective.

As seen from the examples so far $[30,46]$, SAR systems without social awareness can cause problems in human environments. Following human societal social norms may help robots be more accepted in homes, hospitals, and workplaces. Robots should not treat humans as dynamic objects through their navigation behavior, as shown in our results in Figure 1. The figure illustrates a comparison between a traditional planner (green trajectory) that optimizes performance (time, distance, etc.) and a socially aware planner (blue trajectory), which optimizes for social norms along with performance metrics. The traditional planner treats both humans and objects the same way (not maintaining enough distance around humans and objects); however, that is not acceptable. It is acceptable to get close to an object, but a similar behavior around a person is not acceptable. 


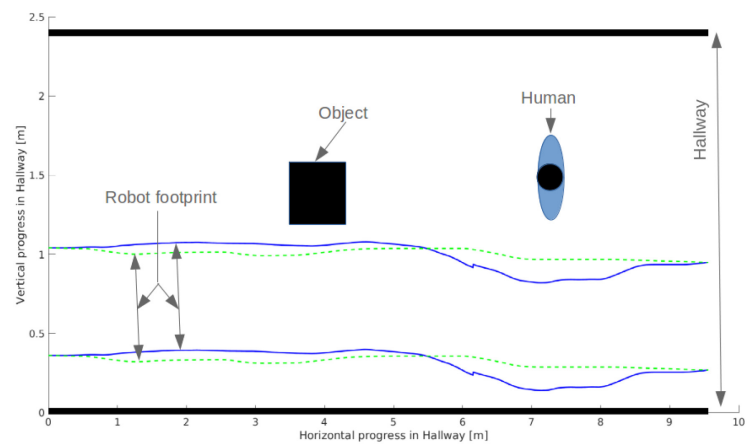

Fig. 1. The PaCcET local planner (blue, solid) compared with the traditional ROS local planner (green, dotted), which does not account for social norms. The traditional planner generated a trajectory that is close to both human and the object (black box), treating them alike. Our approach, the PaCcET-based SAN planner, generated a trajectory that diverged around the human, thereby respecting the personal space of the human.

Socially Aware Navigation planners, including our method, considers the theory of proxemics and other social norms such that the robot does not invade the space around the human. Proxemics [28] codifies this notion of personal space; researchers interested in socially aware navigation (SAN) are investigating methods to integrate the rules of proxemics into robot navigation behavior.

Our approach to SAN using PaCcET addresses the following limitations of the existing approaches:

(1) Many current approaches depend on exocentric sensing, limiting the robot's services to a particular environment [20].

(2) Approaches may require a large amount of training data [1, 29, 34, 37, 48].

(3) The environment/scenario is a singleton, i.e., only a hallway, a room, and so on, is considered or considers only an approach behavior or a passing behavior [20].

(4) Planners are optimized for single or few objectives with linear combination or weighted sum $[22,23]$.

This article builds on our previous work from Reference [25] and provides a comprehensive evaluation of our SAN planner in multiple scenarios in both simulations and the real-world. We extend our prior work by implementing our proposed method on a real robot with objectives such as inter-personal distance, adherence to a social goal, activity space, and group proxemics and providing a more diverse set of simulated environments and real-world scenarios for evaluation. The remainder of this article is structured as follows: We implemented our proposed SAN method on both holonomic and non-holonomic platforms. In the next section, we review related works. In Section 3, we present our approach to SAN using PaCcET. In Section 4, we apply our method to various scenarios in simulation and on a pioneer-3DX platform to validate the proposed approach. Finally, in Section 5, we discuss our present and ongoing work.

\section{RELATED WORK}

\subsection{Socially Aware Navigation}

When localization and navigation were relatively new in the field of robotics, tour-guide robots Rhino and Minerva [11, 12,60] successfully navigated and gave museum tours for visitors. These robots were some of the pioneering works in the field of robot navigation, which performed various navigation functions such as mapping, localization, collision avoidance, and path planning. These robots also exhibited primitive navigation behaviors around people in a dynamic human 
environment. Robust long-term navigation in the indoor environment was demonstrated by Marder et al. [42] when a PR2, a mobile manipulation robot, completed a 26.2-mile run in an office environment. This state-of-the-art navigation technique (traditional planner) can generate a collision-free path and maneuver a robot on that path to get to a goal. However, these algorithms are not sophisticated enough to deal with social interactions while navigating in highly dynamic human environments.

There is a rapidly growing HRI community that is addressing social navigation planners [20, 27, $38,50,54,61]$. Most of the SAN research can be broadly classified (based on areas concerning social navigation) into Planning, Perception, and Behavior Selection. Most of the work is concentrated in the planning area (in turn classified into local planning and global planning). The solutions to SANassociated problems range from simple cost functions to more advanced deep neural networks; we present some of them here.

Social Force Model (SFM) [31] is one of the popular approaches to SAN, which mimics human navigation behavior. Building upon prior work, Ferrer et al. programmed a robot to obey the social forces during navigation activities $[22,23]$. The method also extends SFM to allow a robot to accompany a human while providing a method for learning the model parameters. Kivrak et al. [35] also adopted SFM to be used as a local planner to generate a socially aware trajectory in a hallway scenario. Silva et al. [56] took a shared effort approach to solve the human-robot collision avoidance problem using Reinforcement Learning. Simulated results show that the approach enabled the agents (human and robot) to avoid collisions mutually. Our proposed approach is validated not in a single context, but multiple contexts such as hallway interactions, joining a group of people, waiting in a line, and an art gallery interaction.

Dondrup et al. [17] proposed a combination of well-known sample-based planning and velocity costmaps to achieve socially aware navigation. The authors used a Bayesian temporal model to represent the navigation intent of robot and human based on Qualitative Trajectory Calculus and used these descriptors as constraints for trajectory generation. Alonso et al. [2] presented an $\epsilon$-cooperative collision avoidance method in dynamic environments among interactive agents (robots or humans). The method relies on reciprocal velocity obstacles, given a global path, to compute a collision-free local path for a short duration. Turnwald et al. [62] presented a gametheoretic approach to SAN utilizing concepts from non-cooperative games and Nash equilibrium. This game theory-based SAN planner was evaluated against established planners such as reciprocal velocity obstacles or social forces, and a variation of the Turing test was administered, which determines whether participants can differentiate between human motions and artificially generated motions. Aroor et al. [3] formulated a Bayesian approach to develop an online global crowd model using a laser scanner. The model uses two new algorithms, CUSUM-A* (to track the spatiotemporal changes) and Risk- $A^{*}$ (to adjust for navigation cost due to interactions with humans), that rely on local observation to continuously update the crowd model. Unlike other model-based approaches, our method does not require any training data to perform socially aware navigation.

Okal et al. [48] presented a Bayesian Inverse Reinforcement Learning (BIRL)-based approach to achieving socially normative robot navigation using expert demonstrations. The method extends BIRL to include a flexible graph-based representation to capture the relevant task structure that relies on collections of sampled trajectories. Kim et al. [34] presented an Inverse Reinforcement Learning (IRL)-based framework for socially adaptive path planning to generate human-like trajectories in dynamic human environments. The framework consists of three modules: a feature extractor, a learning module, and a path planning module. Kretzschmar et al. [37] proposed a method to learn policies from demonstrations to learn the model parameters of cooperative human navigation behavior that match the observed behavior concerning 
user-defined features. They used Hamiltonian Markov chain Monte Carlo sampling to compute the feature expectations. To adequately explore the space of trajectories, the method relied on the Voronoi graph of the environment from start to target position of the robot.

Human motion prediction is vital in SAN, as it allows the robot to plan and execute its motion behaviors according to the predicted human motion. In contrast to traditional human trajectory prediction approaches that use hand-crafted functions (social forces), Alahi et al. [1] proposed an LSTM (Long Short-Term Memory) model that can observe general human motion and predict their future trajectories. Hamandi et al. [29] developed a novel approach using deep learning (LSTM) called DeepMoTIon, trained over public pedestrian surveillance data to predict human velocities. The DeepMoTIon method used a trained model to achieve human-aware navigation, where the robots imitate humans to navigate in crowded environments safely.

Although SAN research is dominated by planning-related advancements, for a long-term HRI in human environments, we need to understand how cooperative human navigation works. Psychologists and roboticists are looking into social cues and their effects on HRI to better understand the socially aware navigation problem. Suvei et al. [57] investigated the problem of "how a robot can get closer to people that it wants to interact with?" In a $2 \times 2$ between-subject study, the authors investigated the effect of social gaze cues on the personal space invasion using a human-sized mobile robot. The results from a $2 \times 2$ between-subjects experiment, with/without personal space invasion and with/without a social gaze cue, indicate that social gaze did play a role in participants' perceived safety of the robot. In another study, Tan et al. showed that bystanders and observers of HRI felt safer around the robot than the actual interaction partner even though they both were in very close proximity to the robot [59]. The authors justify the robot's design by collecting the responses of invited users evaluating the properties and appearance of the robot while interacting with it. Rajamohan et al. [49], studied the role of robot height in HRI related to preferred interaction distance. Subjective data showed that participants regarded robots more favorably following their participation in the study. Moreover, participants rated the NAO most positively and the PR2 (Tall, with a fully expanded telescoping spine) most negatively.

\subsection{Multi-objective Optimization}

It is easy to think of a task as a single objective function, where there is a goal or cost function that we are trying to either minimize or maximize. Ideally, this would always give the optimal solution for a task; however, this is not always the case. More often than not, multiple variables contribute to a cost function. An example of this is from basic economics, where there exists a market for a widget. As the supply of this widget goes up, the demand decreases and vice versa. This phenomenon would be known as a supply and demand curve where one objective is the supply, and the other is the demand. In this case, the seller would want to find the optimal amount of supply, such that demand provides the optimal amount of profit. If graphed, this supply and demand curve, as shown in Figure 2, the points on the line would be Pareto-optimal points; no point dominates any other. In this case, the seller is trying to maximize both objectives; therefore, the hollow circles are the dominated points, as there exist solid circle points that are better in both objectives. Typically, multiple Pareto-optimal points are forming a set, which is the solution type that many multi-objective algorithms use [15].

Multi-objective optimization has already started to play a role in real-world applications [43]. Some examples of real-world multi-objective scenarios are high-speed civilian aircraft transportation [44], urban planning [4], and for designing trusses [14]. The trajectory planner used in this article builds off of a pre-existing one that utilizes a multi-objective approach along with linear combination [42]. 


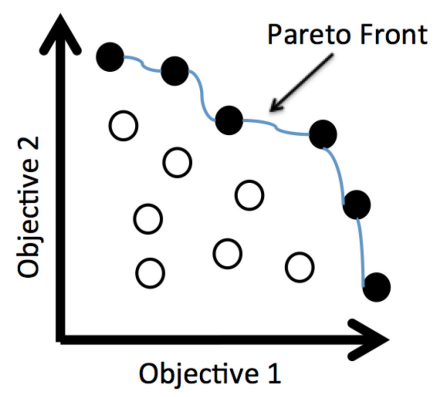

Fig. 2. Multi-objective solution space - The Pareto front contains the non-dominated solutions based on the the two objectives.

2.2.1 PaCcET. In some cases, optimizing a single objective does not yield the desired performance, and therefore multiple objectives need to be considered when evaluating a policy's fitness. A standard method is to multiply a preset scalar value to each objective's fitness score and then add them all together. In some domains, this can lead to an optimal set of policies; however, in some complicated domains, this method will yield sub-optimal policies. A solution to this is to use a multi-objective tool, such as PaCcET, to evaluate policies on multiple objectives [64,65] properly. PaCcET works by first obtaining an understanding of the solution space and finding the Paretooptimal solutions. Next, PaCcET transforms the solution space and then compares each solution giving a single fitness score representative of how well each solution performed in the transformed space.

At a high level, PaCcET works by transforming the Pareto front in the objective space in a way that it is forced to be convex. Transforming to objective space allows the linear combination of transformed objectives to find a new Pareto-optimal point. PaCcET iteratively updates this transformation to force non-explored areas of the Pareto front to be more highly valued than points dominated by the Pareto front or points that are on the explored areas of the Pareto front.

PaCcET has seen a variety of applications: It has been used to extend the life of a fuel cell in a hybrid turbine-fuel cell power generation system [16], the operation of the electrical grid on naval vessels [52], the coordination of multi-robot systems [63], and for the efficient operation of a distributed electrical microgrid [53], where a series of small power generation systems coordinate to meet the demands of consumers. In each of these applications, it has been shown that PaCcET functions at or above the solution quality of other techniques such as NSGA-II or SPEA2 [64], with as low as one-tenth of the runtime.

For this project, $\mathrm{PaCcET}$ was used over other multi-objective tools because of its computational speed [64], as shown in Figure 3. PaCcET was used to evaluate the possible trajectories developed in the local planner. At each timestep, the sensor data is analyzed, and the desired features are evaluated for each of the potential future trajectory points. PaCcET then uses the fitness values for each feature of every future trajectory to develop the solution space and obtain the optimal future trajectory. Since, at each timestep, future trajectories are developed independently, PaCcET develops a brand new solution space at each timestep. By developing a new solution space at each timestep, the local planner can be optimized in real-time.

\section{METHOD}

In this section, we detail our methodology of a socially aware navigation planner, the features or objectives that we used to optimize the trajectories, and how PaCcET was implemented in the local trajectory selection process. Figure 4 shows the overall high-level block diagram of the proposed 


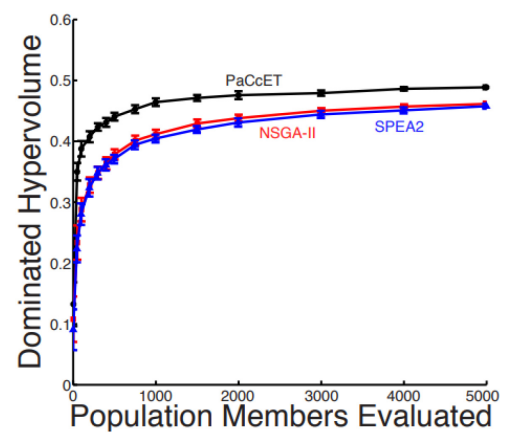

Fig. 3. PaCcET computational speed-Percentage of hypervolume dominated in Kursawe's (KUR) problem in comparison with two successful multi-objective methods, SPEA2 and NSGA-II. This plot shows that PaCcET proceeds faster towards the Pareto front.

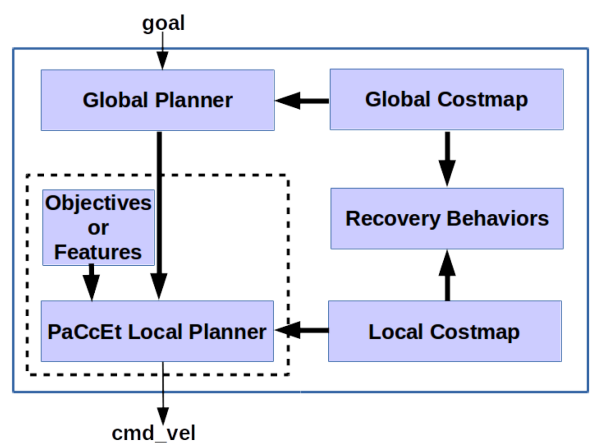

Fig. 4. Block diagram of the proposed approach, a modification of ROS navigation stack's local planner using PaCcET-based non-linear optimization.

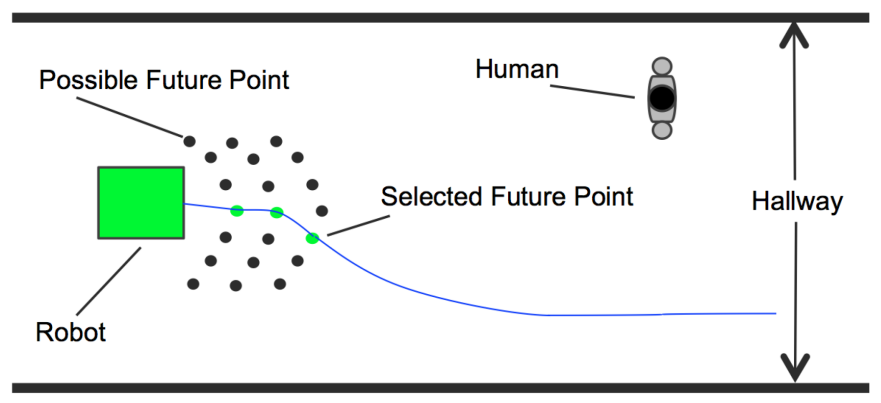

Fig. 5. Navigation Planner - The navigation planner selects a short-term trajectory (green points represent potential trajectory end-points) from the pool of possible trajectories (black points), optimized for adherence to a long-term plan (blue line), obstacle avoidance, and progress toward a goal, and in the case of this article, interpersonal distance.

approach. It is built on top of the well-established ROS navigation stack by modifying the local planner using PaCcET. The overall function of the local trajectory planner at each timestep is to generate an array of possible future trajectory points and evaluate each future trajectory point based on a predefined feature set as shown in Figure 5. In previous work, the features were assumed to have either no relationship or a simple linear relationship with one another; however, this is 
not always the case. Therefore, we need to consider the possibility that the features are dependent on each other and have nonlinear relationships.

\subsection{Features/Objectives}

In the traditional navigation planner, the features extracted were each assigned their own cost (e.g., the path distance cost $\left(\Delta_{p a t h}\right)$, the length that the robot has already traveled, the goal distance cost $\left(\Delta_{\text {goal }}\right)$, the distance the robot is from the goal) [42]. The path distance will have a linear relationship with the goal distance, since the change in one has a direct linear impact on the other. Once each feature has a cost associated with it, each cost is multiplied by a pre-tuned scalar and then added together, thus giving a linear combination, or weighted sum, in this case, the cost function shown in Equation (1). We can think of this cost function as an objective, where each possible future trajectory point has a cost or fitness associated with that objective. Since the purpose is to minimize the overall cost function, the planner will take the best path possible that minimizes the function, which in this case, will minimize both features.

$$
\operatorname{cost}\left(v_{x}, v_{y}, v_{\theta}\right)=\alpha\left(\Delta_{\text {path }}\right)+\beta\left(\Delta_{\text {goal }}\right)
$$

This cost function has been adapted to include a heading difference $\left(\Delta_{\text {heading }}\right)$ feature and an occupancy $\left(\Delta_{o c c}\right)$ cost feature, where the heading difference is the distance that the robot is from the global path and the occupancy cost is the cost used to keep the robot from hitting something. The same approach as in the previous cost function is taken in Equation (2). By taking a closer look at just the heading difference and how that might affect the path distance or the goal distance, it becomes less clear if there is only a linear relationship between the four. For example, if there is an obstacle in the robot's path, it will try and minimize goal distance by changing its heading, thus increasing the heading feature cost. In turn, this also increases the path distance cost, though this may or may not be linear.

$$
\begin{aligned}
\operatorname{cost}\left(v_{x}, v_{y}, v_{\theta}\right)= & \alpha\left(\Delta_{\text {path }}\right)+\beta\left(\Delta_{\text {goal }}\right)+\gamma\left(\Delta_{\text {heading }}\right) \\
& +\delta\left(\Delta_{\text {occ }}\right)
\end{aligned}
$$

Building upon prior work done in this area, we include socially aware navigation features such as interpersonal distance (ID), distance from a group $(G D)$, and distance from a social goal $(S G D)$. As a way to dissuade the robot from getting too close to a human, a cost function was developed to penalize the robot at an exponential rate as the interpersonal distance decreases, as seen in Equation (3) (for every human in the interaction scenario). Although we could penalize the robot based on this at all times, it is not necessary if the interpersonal distance is so significant that it would not be considered as a socially inappropriate distance. Therefore, the robot is only penalized if the interpersonal distance is less than or equal to 1.5 meters. The interpersonal distance threshold was chosen to be 1.5 meters to ensure that the robot remains in social space and does not invade the personal space of the person [28].

$$
I D_{f}=e^{1 / I D}
$$

For the robot to not get too close to a group of people or not get in between them, we penalized the robot based on $G D$ whenever it is close to a group using Equation (4).

$$
G D_{f}=e^{1 / G D}
$$

Contrary to Equations (3) and (4), Equation (5) is akin to a reward to get closer to a social goal rather than an actual goal. With this feature in place, the robot tends to reach a social goal for a particular scenario while still adhering to the final goal location. For example, the social goal for reaching the front of a desk when others are waiting in a line is the end of the line. So, the 
robot will reach the social goal first (end of the line) and eventually reaches the desk when it is the robot's turn.

$$
S G D_{f}=e^{S G D}
$$

Instead of adding these features' cost into the previous cost function, as in Equation (2), we assume that its relationship with other features might be nonlinear and therefore gets treated as separate objectives. Since we know that the above cost function, Equation (2), works sufficiently enough from previous work [42], we can treat it as its objective as well. Instead of optimizing just one objective, we need to optimize multiple objectives, hence our multi-objective approach. Using a multi-objective tool like PaCcET requires computational time, and, since this is intended to work in real-time, any chance to improve the computation time should be utilized. Treating the first four features used in the previous cost equation as a single objective not only speeds up this process but, in turn, allows for the possibility to add even more features to our local trajectory planner. Using $\mathrm{PaCcET}$ to do the multi-objective transformations, we essentially get a new cost function with a $\mathrm{PaCcET}$ fitness denoted by $P_{f}$, which was modeled under the assumption of nonlinear relationships between the objectives. Equation (6) shows how $P_{f}$ is a transformation function dependent on multiple variables.

$$
P_{f}=T_{f}\left(O b j_{1}, \mathrm{Obj}_{2}, \ldots, O b j_{n}\right)
$$

In this work, we are only interested in objectives such as interpersonal distance, distance from a group, and distance from the social goal. The first objective is the original cost function (Equation (2)), which is the linear combination of the path distance, goal distance, heading difference, and occupancy cost. The remaining objectives are the social features, such as: interpersonal distance, distance from social goal, and so on. Equation (7) shows the PaCcET fitness function with our proposed objectives.

$$
P_{f}=T_{f}\left(\cos t\left(v_{x}, v_{y}, v_{\theta}\right), I D_{f 1}, \ldots, I D_{f n}, G D_{f}, S G D_{f}\right),
$$

where $I D_{f 1}, . . I D_{f n}$ are the cost functions associated with interpersonal distance between $n$ people and the robot.

\subsection{Trajectory Planning}

The robot's trajectory planning algorithm can be broken into three parts: the global planner, the local planner, and low-level collision detection and avoidance. The global trajectory planner works by using knowledge of the map to produce an optimal route given the robot's starting position and the goal position. The global path is created as a high-level planning task based upon the robot's existing map of the environment; this is regenerated every few seconds to take advantage of shorter paths that might be found or to navigate around unplanned obstacles. The traditional local planner's role is to stay in line with the global path unless an obstacle makes it deviate from the global path. The low-level collision detector works by stopping the robot if it gets too close to an object. We use the traditional global trajectory planner and low-level collision detector [42] and make adaptations to the local trajectory planner to incorporate interpersonal distance and PaCcET.

Algorithm 1, Algorithm 2, and Algorithm 3 can be summarized as follows:

(1) Discretely sample the robot control space.

(2) For each sampled velocity $\left(V_{x}, V_{y}\right.$, and $\left.V_{\theta}\right)$, perform a forward simulation from the robot's current state for a short duration to see what would happen if the sampled velocities were applied. This is robot-specific, based on the footprint of the robot.

(3) Score the trajectories based on metrics. 
(a) Score each trajectory from the previous step for metrics such as distance to obstacles, distance to a goal, and so on. Discard all the trajectories that lead to a collision in the environment.

(b) For each of the valid trajectories, calculate the social objective fitness scores such as interpersonal distance and other social features and store all the valid trajectories.

(4) Perform Pareto Concavity Elimination Transformation (PaCcET) on the stored trajectories to get a PaCcET fitness score and sort the trajectories from lowest to highest PaCcET fitness score.

(5) For a given timestep, grab the trajectory with index 0 from the sorted list of valid trajectories.

Algorithm 1 shows the primary functions of the local trajectory planner and how the future trajectory points were stored to be used with PaCcET. The trajectory planner is called every timestep, which in this case, is every 0.1 second. Once the trajectory planner is called, the Transform_Human_State function is called to transform human pose to the robot's odometry reference frame, which allows the interpersonal distance corresponding to each possible trajectory to be calculated in the Generate_Trajectory function. There are two methods of calculating the possible trajectories. The first is assuming that the robot can only move forward, backward, and turn. To produce the possible trajectories for this physical setup, we loop through every combination of a sample of linear velocities $\left(V_{x}\right)$ and angular velocities $\left(V_{\theta}\right)$ to generate trajectories. (For a holonomic robot, a slight change in $V_{y}$ is also used to generate possible trajectories.) Once a trajectory is created, we determine if it is valid based on the constraints for the first objective. For example, trajectories that would make the robot hit a wall, obstacle, or human are not considered strong trajectories and, therefore, will not be stored in the Store_Trajectory function. By not storing these invalid trajectories, the speed at which PaCcET runs can be improved.

The second method assumes that the robot is capable of holonomic movement and can translate with any $V_{x}, V_{y}, V_{\theta}$ that are less than velocity limits. Given these movements, we again loop through all the possible movements given the predefined number of $V_{x}$ samples, $V_{y}$ samples, and $V_{\theta}$ samples. Again, if the trajectories are valid, then they are stored. Once all the valid trajectories are stored for all possible movements, the Run_PaCcET function runs, giving back the best possible trajectory, $\left(\mathcal{T}_{\mathcal{B}}\right)$, based on its multi-objective transformation process.

To run a multi-objective tool like PaCcET, each objective's fitness needs to be calculated. Algorithm 2 details the Generate_Trajectory function from Algorithm 1. The first function that needs to be performed is the Calculate_State function, as the robot's position and velocity are used to determine the fitness values for the objectives. Using the state information the Compute_Path_Dist, Compute_Goal_Dist, Compute_Occ_Cost, and Compute_Heading_Diff functions are used to calculate the fitness values associated with the four pieces of the first objective. Using those fitness values, the first objective's fitness is calculated by the Compute_Cost function. Distance-based features such as interpersonal distance of each person, group distance, and social goal distance are calculated, as shown in Algorithm 2 lines 8-10. Once all the objectives have their fitness values, the trajectory along with the fitness values is returned to the local trajectory planner algorithm, which saves all the valid trajectories and calls PaCcET Algorithm 3 to output socially appropriate trajectories.

\subsection{Integrating PaCcET}

At the end of Algorithm 1, all the valid trajectories have been stored along with their objective fitness scores in a vector of type trajectory. Algorithm 3 details the primary functions for determining a single fitness value from multiple objectives. To run PaCcET, the objectives for 


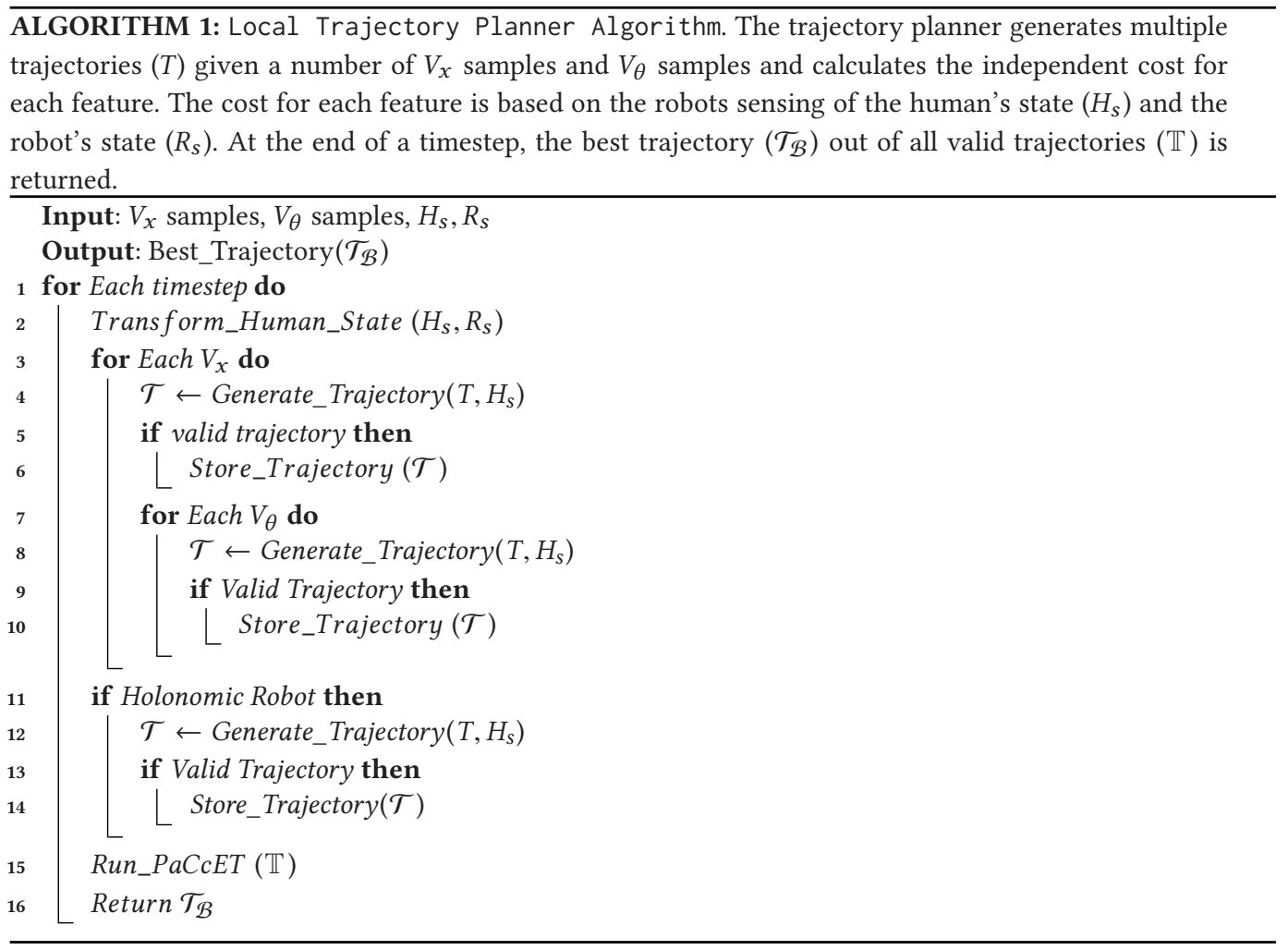

each trajectory must be stored in a vector of type double, which is done in the Store_Objectives function. Before running PaCcET's primary functions, an instance of PaCcET must be created. Next, the solution space and Pareto front are created by giving each trajectory to the Pareto_Check function. Now that the Pareto front and its geometry have been calculated, $\mathrm{PaCcET}$ can transform the solution space and give a single fitness value for each trajectory in the Compute_PaCcET_Fitness function. Once each trajectory has its PaCcET fitness, they are sorted from best to worst in the Sort_Trajectories function, which allows the function to not only ascertain the best trajectory easily, but is also useful for debugging purposes. Algorithm 3 concludes by returning the best trajectory to the local trajectory planner algorithm.

\subsection{Social Goal}

Computing the social goal location is important, because often the actual goal location may not be an appropriate location for interaction and explicitly commanding the social goal would not be possible. A social goal can be defined as an appropriate location for a robot to involve in humanrobot interaction. For example, in a front desk-like scenario, the end of the line can be considered social. For this work, the social goals for each interaction scenario were geometrically computed, for $O$-formation scenario (joining a group), we fit a circle with the people in a group and find a socially appropriate spot to join the group, as shown in Figure 6.

We found angles made by every person with the center of the formed circle using the law of cosines: Equation (8), as shown below:

$$
c^{2}=a^{2}+b^{2}-2 a b * \cos (\theta),
$$




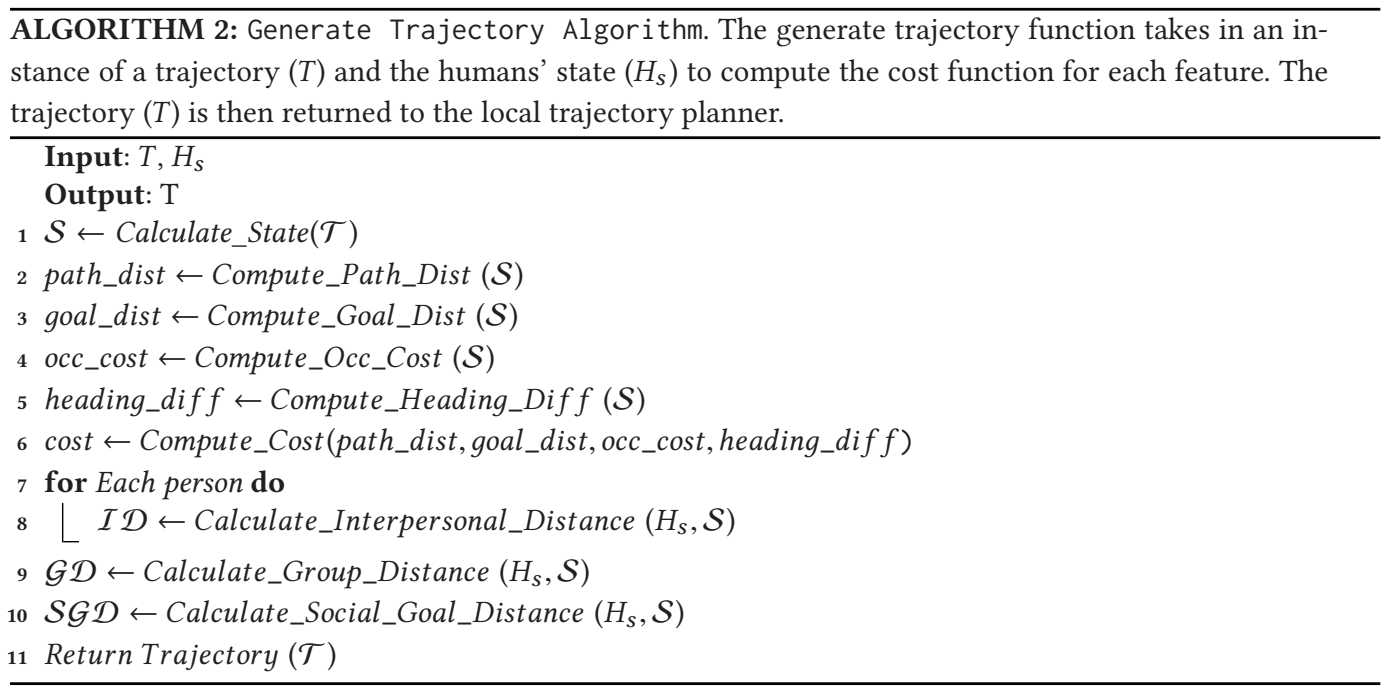

ALGORITHM 3: PaCcET Alogrithm. PaCcET $(P)$, takes in the vector of valid possible trajectories $\mathbb{T}$ to compute the multi-objective space and the PaCcET fitness $\left(P_{f}\right)$ for each trajectory.

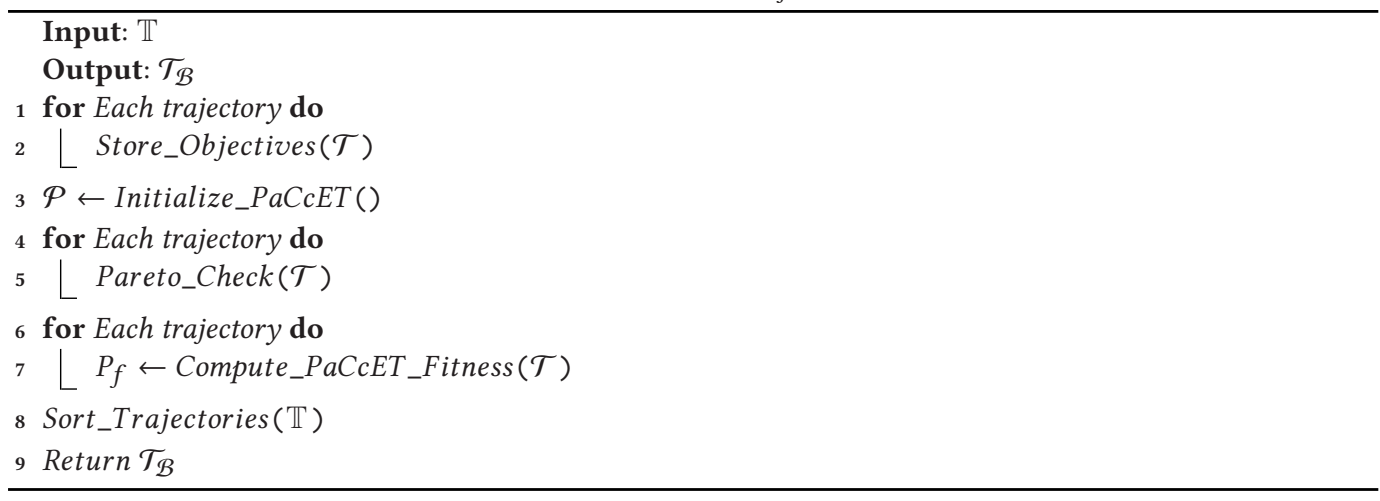

$$
\theta_{i j}=\cos ^{-1}\left[\left(2 R^{2}-D_{i j}\right) / 2 R^{2}\right]
$$

where $D_{i j}$ is the Euclidean distance between person $i, j$, and $R$ is the radius of the circle formed by all the people in the group. Out of all the $\theta_{i j}$ 's, we pick one half of the widest angle as approach angle denoted by $\theta_{a}$. Now, joining a group problem (O-formation) boils doing to finding the intersection of two circles, one formed by the people in the group and the other formed in the wide-open sector with the center as the locations of either of the people making the widest sector. The equations of the two circles to solve for are given as follows:

$$
\begin{gathered}
(x-h)^{2}+(y-k)^{2}=R^{2}, \\
\left(x-h_{p}\right)^{2}+\left(y-k_{p}\right)^{2}=r^{2},
\end{gathered}
$$

where $(h, k)$ is the center of the circle formed by the group of people, $\left(h_{p}, k_{p}\right)$ is the location of one of the persons that formed the widest sector. The radius $r$ in Equation (11) is obtained by solving 


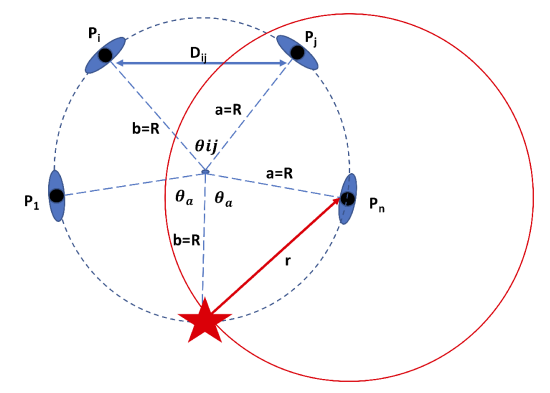

Fig. 6. Figure illustrating the computation of social goal in the O-formation scenario. The red star represents a goal that respects social norms.

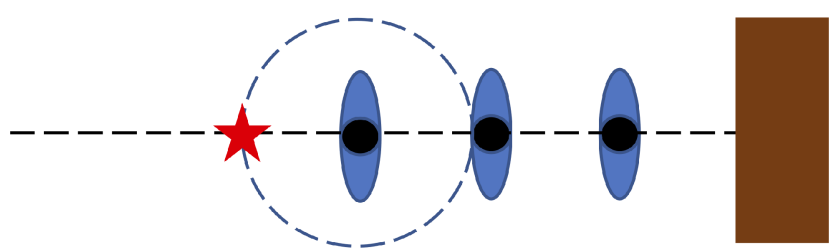

Fig. 7. Figure illustrating the computation of social goal in waiting in a queue scenario. The red star represents the social goal.

for $c$ in Equation (8), where $\theta=\theta_{a}, a$ and $b$ equals $R$, radius of the group formation. There are two solutions when solving Equations (10) and (11), and we further filter one social goal from the two solutions.

Similarly, we can fit a straight line as shown in Figure 7 for waiting in a queue scenario, and social goal location would be the end of the line considering the personal space of the last person in the line. Hence, in this case, the solution boils down to solving for the intersection of a line and a circle. The equation of the line formed by the people can be found by fitting a line of form $y=m x+c$ with the people's locations. The circle formed using the last person's location as the center and a comfortable distance that the robot should maintain around the last person as the radius is of the form $(x-k)^{2}+(y-h)^{2}=r^{2}$. The two solutions to the line and circle intersection can be obtained using quadratic roots, and the social goal is further filtered to the solution farthest to the actual goal (desk).

The social goal calculation for Scenario 2 (art gallery) is hand-selected. Computing the social goal in this scenario is beyond the scope of this article. The art-gallery scenario requires a perception pipeline that can detect the location of the art on display, the area, and perimeter of the artwork to compute a useful social goal location to interact with a person viewing the art or to determine the activity zone to avoid traversing it. The social goal for Scenario 2 will be addressed in our ongoing work on USAN; see Section 5.

In this section, we presented an in-depth illustration of our proposed PaCcET-based social planner, various cardinal objectives related to SAN, and methods to determine social goals using spatial information such as locations of people. The next section, Section 4, presents the results of robots performing appropriate navigation behaviors in simulation and real-world environments.

\section{RESULTS}

To validate our proposed approach, we considered four different scenarios, namely, a hallway scenario, an art gallery scenario, forming a group, and waiting in a queue. All this was tested in 


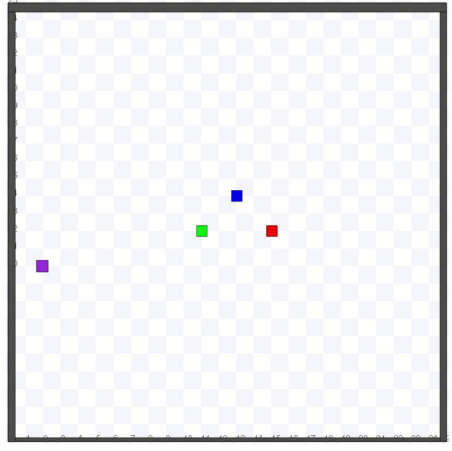

(a) Stage, a 2D simulator.

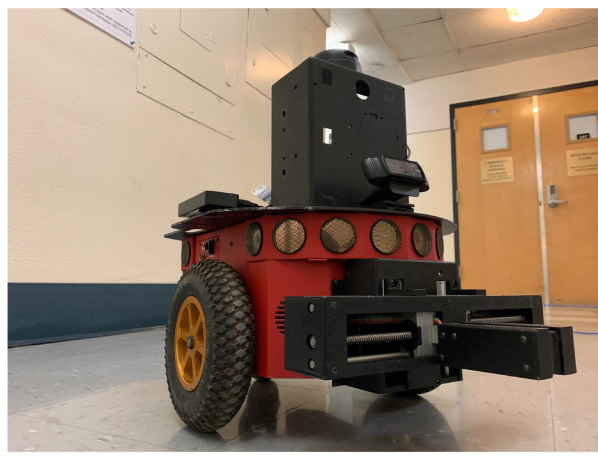

(b) Upgraded Pioneer 3DX robot.

Fig. 8. Platforms used to validate our proposed social planner.

simulation using the 2D simulator, Stage [26] on a machine with an Intel sixth-generation i7 processor @3.4 GHz, 32 GB of RAM. The simulated environment for each hallway experiment was the second-floor hallway of the Scrugham Engineering and Mines building at the University of Nevada, Reno. The map of the building used in the simulation was built using the gmapping package for SLAM on the PR2. The simulated PR2 is comparable to the real-world PR2 for sensing and movement capabilities and is using AMCL for localization on the map. The simulated PR2 uses a 30-meter range laser scanner that is identical to the real PR2 robot's laser scanner's capability and the humans in the simulation exhibit very simple motion behaviors. Follow-up scenarios are simulated in a $25 \mathrm{~m} \times 25 \mathrm{~m}$ open space in the stage environment, as shown in Figure 8 (a). PR2 robot was simulated to run both traditional planner and our modified PaCcET based planner. In Figure 8(a), the purple agent is the simulated PR2, and the rest of the agents are humans formed as a group. For real-world validation, we used an upgraded Pioneer 3DX platform, shown in Figure 8(b). The Pioneer robot that we used is equipped with an RPLIDAR-A3, a 30-meter range laser scanner with a $360^{\circ}$ field of view, and a webcam as sensors for perception. For detecting people using a laser scanner, we used the work of Leigh et al. [40]. The robot's computational unit is also upgraded to a laptop with an Intel Core i7-7700HQ CPU @ $2.80 \mathrm{GHz} \times 8$ processors, 16 GB RAM GeForce GTX 1050 Ti GPU with 4 GB of memory. The pioneer robot also uses AMCL for localization on the map. The hallway scenarios on the real robot were validated in the same location as the simulation experiments. Art gallery, waiting in line, and group formation scenarios are validated in the lobby area $(7 \mathrm{~m} \times 7 \mathrm{~m}$ approx.) situated on the first floor of the Scrugham Engineering and Mines building of University of Nevada, Reno.

\subsection{Simple Scenario}

The hallway scenario was divided into four sub-scenarios, namely, passing a stationary human, passing a walking human in the same direction, an encounter with a human walking on the appropriate side, and passing a walking human in the opposite direction.

In the first experiment, the simulated robot was tasked with getting to a goal while passing close to a static simulated human. Figure 9(a) shows that when using the traditional planner, the robot made sure to avoid a collision with the simulated human but did not consider any social distance. The same will be the case for the other experiments as well, since the traditional planner does not consider interpersonal distance into its cost function. The PaCcET-based planner did consider interpersonal distance, and therefore the robot deviated from a more straight-lined path as a way to satisfy the second objective. Once the threshold for the interpersonal distance was no longer an 


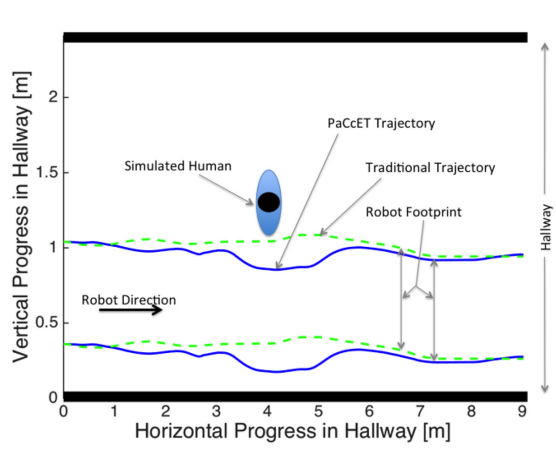

(a) Scenario 1: Simulated PR2 passing a simulated stationary human in a narrow hallway.

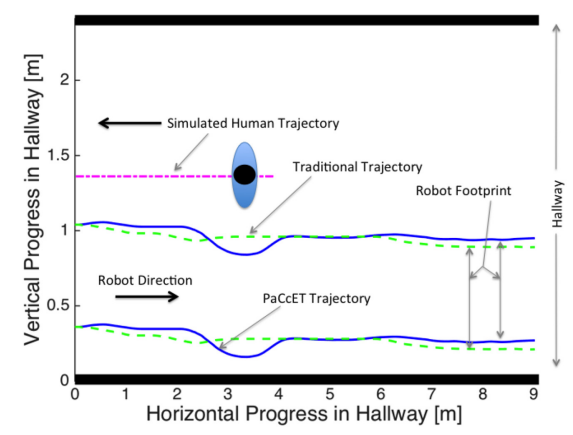

(c) Scenario 1: Simulated PR2 encounters a simulated human passing on the appropriate side of a narrow hallway.

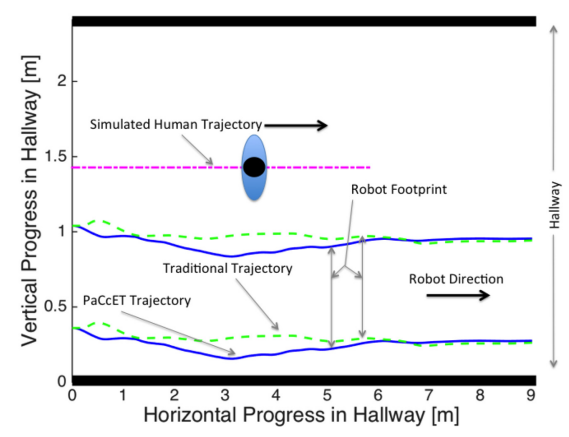

(b) Scenario 1: Simulated PR2 passing a simulated human walking in the same direction as the PR2 in a narrow hallway.

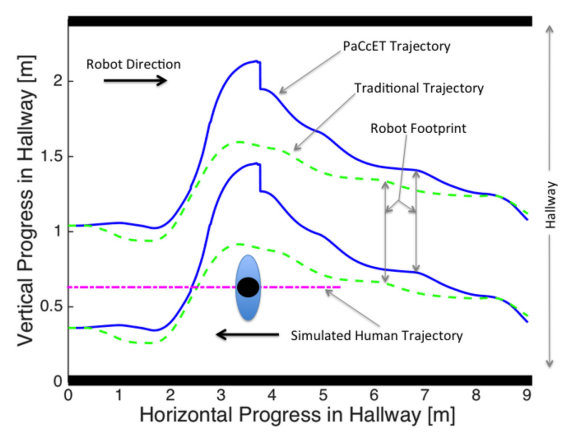

(d) Scenario 1: Simulated PR2 encounters a simulated human walking on the inappropriate side of a narrow hallway in opposite direction.

Fig. 9. Simple two-objective optimization scenarios with a single simulated human. The simulated human trajectory is shown using a dotted magenta line, trajectory of traditional planner is represented using a dotted green lines (two lines to represent footprint of the simulated PR2) and PaCcET-based SAN trajectory is represented using a solid blue line (two lines to represent footprint of the simulated PR2). Direction of simulated human and PR2 are represented using arrows.

issue, the robot only needed to minimize the first objective; therefore, returning to a straight-line path. It is worth noting that in all the conducted experiments, the robot also considered a wall as an obstacle and was required to disregard trajectories that would lead to a collision, which is why the robot refrained from deviating any further from the global trajectory.

The second experiment was developed to mimic a passing scenario where the robot has a set goal but needs to pass by a simulated human who is traveling much slower in the same direction. Figure 9(b) shows that the traditional trajectory planner merely made sure that a collision would not take place as it tried to minimize its cost function. The PaCcET-based planner deviated from its global trajectory to consider the interpersonal distance objective, then returned to the global trajectory once the threshold for the interpersonal distance was no longer an issue.

Similar to the previous experiment, the third experiment involves both the simulated human and robot moving; however, in this case, the simulated human is now moving at a normal walking speed in the opposite direction of the robot-the robot and simulated human pass close to one another but not close enough to cause a collision. Figure 9(c) shows that the traditional trajectory 


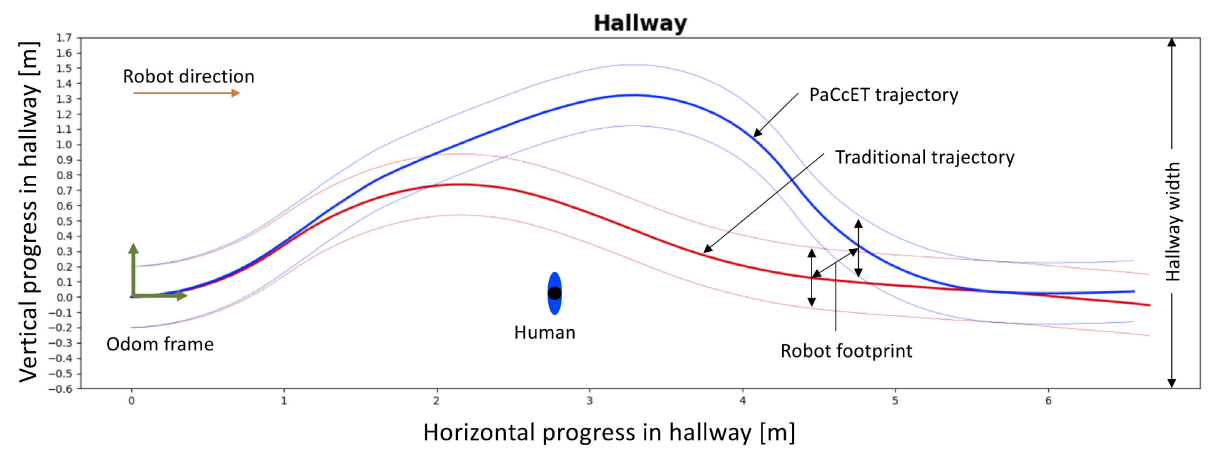

Fig. 10. Scenario 1: (real-world interaction) Pioneer robot encounters a stationary human standing in the path of the robot in a hallway.

planner altered its path ever so slightly to ensure that a collision would not happen, where the PaCcET-based trajectory planner not only ensured that a collision would not take place but also considered interpersonal distance and provided the simulated human with additional space while passing.

The previous experiments show that when using a PaCcET-based trajectory planner, interpersonal distance can be considered when selecting a local trajectory in both static and dynamic conditions where a collision is not imminent. However, the case of a collision that would occur unless either the simulated human or the robot moves out of the way also needs to be considered. This experiment considers a simulated human who is not paying attention or unwilling to change their course and walking directly towards the robot. Figure 9(d) shows that the traditional trajectory planner was successful at avoiding the collision as expected; however, it did so while minimizing its cost function as much as possible, which caused the robot to get very close to the simulated human. When using the PaCcET-based trajectory planner, the robot not only avoided the collision but also gave the simulated human additional space to satisfy the interpersonal distance objective. It is worth noting that once the interpersonal distance threshold was no longer an issue, the robot used its holonomic movement for a short time as a way to quickly minimize the heading difference portion of the original cost function objective.

We extended hallway scenarios to the real-world by implementing our proposed approach on a Pioneer 3DX robot and validating it in both static and dynamic environments. Figure 10 shows a real-world hallway situation where a human is standing in the path of a robot that is attempting to go down the hallway. The robot, when using the traditional planner, treated the human as a mere obstacle and avoided a collision but violated the personal space rule of the human. However, our approach using PaCcET-based local planning considered the stationary human's personal space using interpersonal distance objective and deviated from the global trajectory in such a way that the personal space rule is obeyed. In Figure 10, the blue trajectory is generated by our proposed approach, and the traditional approach generates the red trajectory.

Figure 11 shows a real-world hallway interaction like the previous one, but in this case, the human is moving as opposed to a static human. In this experiment, the human is walking in the opposite direction of the robot and also on the wrong side of the hallway. As one can observe, the traditional planner (red trajectory) managed to avoid a collision with the human but went very close to the person, thereby intruding into the human's personal space. Our proposed approach not only avoided a collision but also maintained a safe distance while trying to avoid the human walking on the wrong side of the hallway. Unlike the PR2, Pioneer is a non-holonomic robot; hence, the holonomic behavior, as seen in Figure 9(d), is not seen in the real-world inter- 


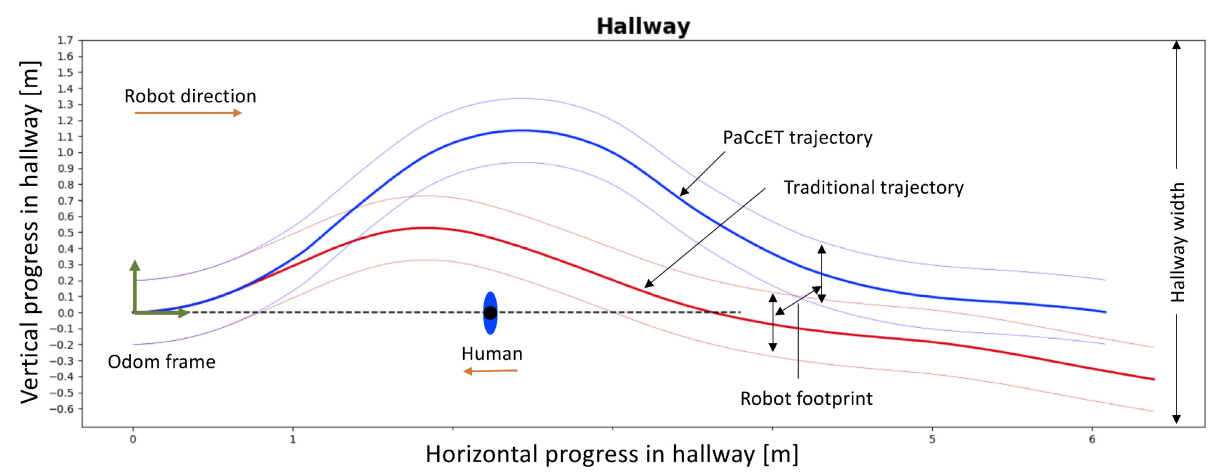

Fig. 11. Scenario 1: (real-world interaction) Pioneer robot encounter a human walking in the opposite direction and on the side of the hallway.

action. It is worth noting that in Figures 10 and 11 the robot with PaCcET trajectory planner showed signs of legibility of movements. In both these cases, the efforts of the robot trying to clear the human's personal space are clearly seen using our method as opposed to the traditional planner.

\subsection{Complex Scenarios}

In the previous section, 4.1, we showed both in simulation and real-world that by just considering one social feature, i.e., interpersonal distance, our approach was able to account for personal space while navigating a hallway (with different maneuvers of a human partner). In this section, we will see the results of our approach applied to complex social scenarios such as art gallery interactions (Figure 12), waiting in a line (Figure 13) and joining a group of people (Figure 14). These scenarios are representative of both human-human and human-environment interactions that occur in normal social discourse.

Figure 12(a) shows the behavior of our social planner and traditional planner in an art gallery situation (three objectives) in simulation. We considered an art-gallery scenario, but it can be generalized to other similar scenarios like a tour-guide robot in a museum or an attraction. For this scenario, we staged a human-robot interaction consisting of a robot presenting a piece of art (hanging to a wall) to a human standing nearby. Both the traditional planner (red line) and the SAN planner (blue line) were given the same goal (represented as a red star) and start (START) locations. The traditional planner steered the robot to the goal location, cutting the standing person from the back (inappropriate). However, the SAN planner steered the robot to a location that is appropriate to present the details of the art to the human (social goal). The SAN planner approached the social goal, leaving enough personal space based on the interpersonal distance feature.

Art-gallery interactions are not always presenting the artwork on display. While navigating an art gallery, one should consider the affordance and activity spaces between the artwork and an individual looking at the art. Activity space is a social space linked to actions performed by agents [41]. For example, the space between the subject and a photographer is an activity space, and we humans generally avoid getting in the way of such activity spaces. Affordance space is defined as a social space related to a potential activity provided by the environment [51]. In other words, affordance spaces are potential activity spaces. An environment like an art gallery provides numerous locations as affordance spaces (place in front of every piece of art is an affordance space). 


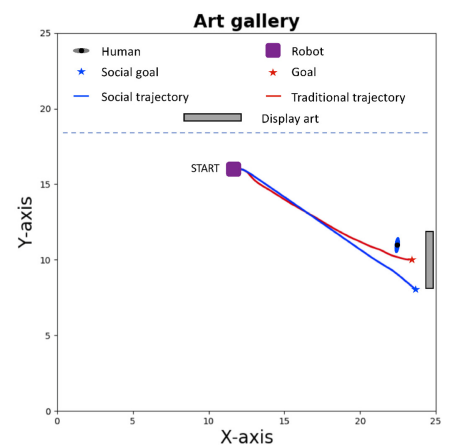

(a) Scenario 2 (simulation): Robot interacting with a human in an art gallery where the robot with SAN planner presents itself at a position appropriate to talk about the art on display, the blue trajectory is generated using the proposed SAN planner.

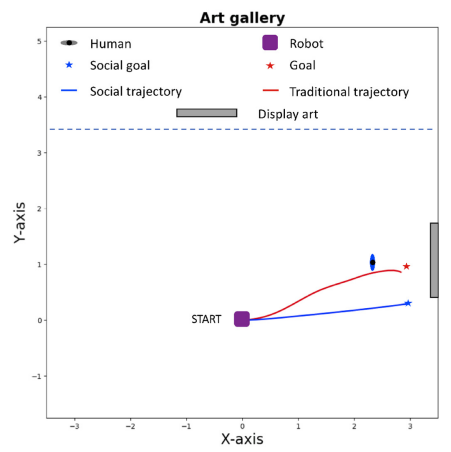

(c) Scenario 2 (real-world): Pioneer robot interacting with a human in an art gallery where the robot with SAN planner presents itself at a position appropriate to talk about the art on display, the blue trajectory is generated using the proposed SAN planner.

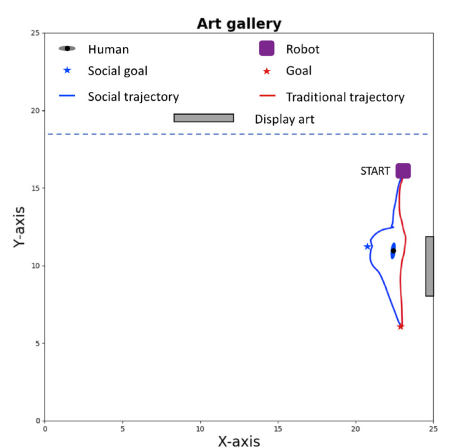

(b) Scenario 2 (simulation): Robot taking onto account activity space in an art gallery where the robot with SAN planner avoids going into the activity space, represented by the blue trajectory.

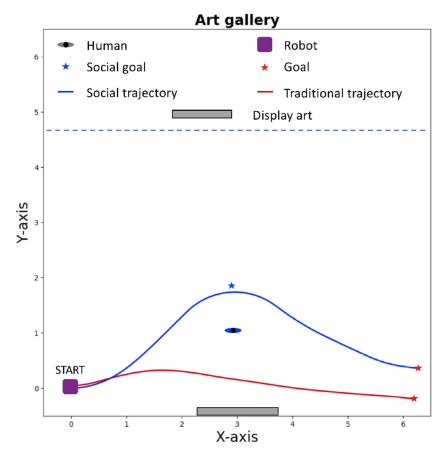

(d) Scenario 2 (real-world): Pioneer robot taking onto account activity space in an art gallery where the robot with SAN planner avoids going into the activity space, represented by the blue trajectory.

Fig. 12. Validation results of Scenario 2 (art gallery) in both simulation and real-world.

When a visitor steps into one such affordance space, that space between the artwork and the interacting human becomes activity space.

In Figure 12(b), we demonstrated an appropriate behavior around activity space in simulation using our proposed SAN planner. For this scenario, we staged a human-robot interaction consisting of a human interacting with a piece of artwork hanging to the wall. Both the traditional planner (red line) and the SAN planner (blue line) were given the same goal (represented as a red star) and start (START) locations. The traditional planner steered the robot to the goal but did not account for the activity space, i.e., the robot traversed through the activity space (inappropriate). However, the PaCcET-based SAN planner steered the robot to the goal location while avoiding the activity space (appropriate social behavior). The social goal, while avoiding an activity zone, is not an end goal where the robot would stop but is more like a social goal that acts as a way-point in reaching the end goal.

Similarly, the two art gallery behaviors (presenting art and avoiding activity space) are implemented and validated on a Pioneer robot, and the results are shown in Figure 12(c) and Figure 12(d) 


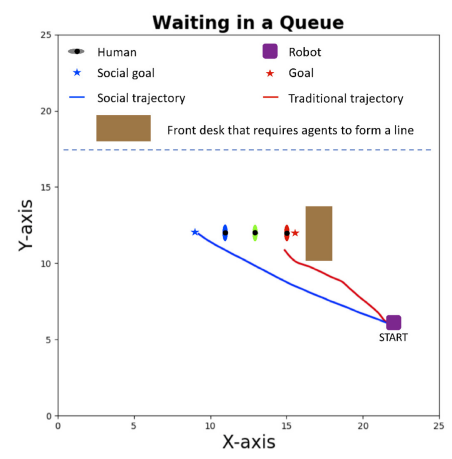

(a) Scenario 3: The robot is joining a line, formed in front of a desk. Traditional planner generated the red trajectory, positioned the robot in an inappropriate location beside the first person while attempting to reach the front of the desk. The blue trajectory is generated using our proposed SAN planner leading the robot to join the line (appropriate).

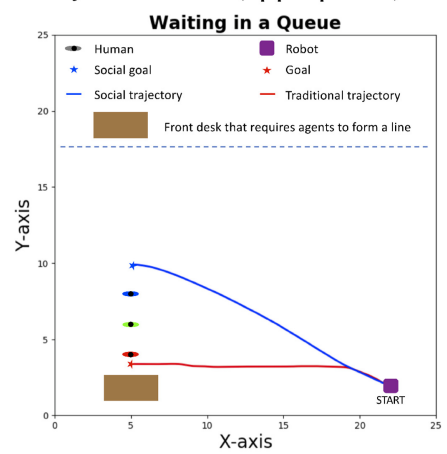

(c) Scenario 3 (location and orientation change): The robot is joining a line, formed in front of a desk scenario. The traditional planner generated the red trajectory, guiding the robot to the front of the desk, cutting the line (inappropriate). The blue trajectory, our proposed approach, leading the robot to join the line (appropriate).

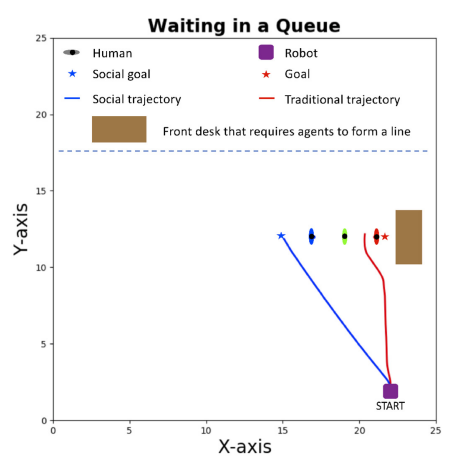

(b) Scenario 3 (location change): The robot is joining a line, formed in front of a desk scenario. The traditional planner generated the red trajectory, guiding the robot between the first two people (inappropriate). The blue trajectory, our proposed approach, leading the robot to join the line (appropriate).

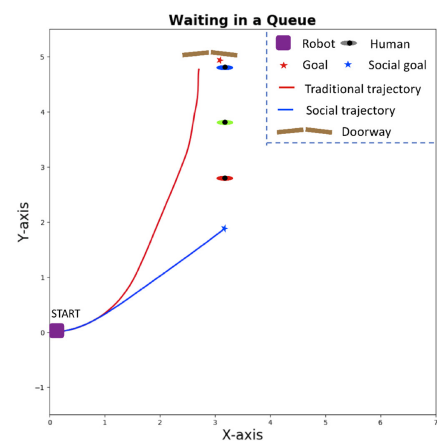

(d) Scenario 3 (real-world): Pioneer robot is joining a line, formed in front of a doorway scenario. The traditional planner generated the red trajectory, guiding the robot to a location besides the first person (inappropriate), cutting the line. The blue trajectory, our proposed approach, leading the robot to join the line (appropriate).

Fig. 13. Validation results of Scenario 3 (waiting in a queue) in both simulation and real-world.

Figure 13(a) shows the behavior of our social planner and traditional planner in the waiting in a queue situation (five objectives) in simulation. Here, we considered a front-desk interaction, but this can be generalized to other similar social scenarios where a robot or a human is required to form a line before reaching the goal. For example, social scenarios such as getting coffee from a public coffee machine, taking an elevator, and so on. In this context, we staged a human-robot interaction consisting of a robot that wants to interact with a front-desk representative of an office building where other people were being served on a first-come-first-served basis. Both the traditional planner (red line) and the SAN planner (blue line) were given the same goal (represented as a red star) and start (START) locations. The trajectory planner tried to steer the robot to the goal location and stopped at an inappropriate location (besides the person currently 
being served) as the traditional planner treated the human as an object. However, the SAN planner steered the robot to an appropriate location, i.e., end of the line, positioning the robot behind the last person (social goal), considering personal space as well.

Figures 13(b) and 13(c) show results with variations in scenario 3 (waiting in a queue). The variations are the locations of people and the orientation of the queue formed by them. Figures 13(b) and 13(c) show that our method is robust. Figure 13(a) shows the behavior of our social planner and traditional planner in the waiting in a queue situation (five objectives) in the real-world. Here, considering a doorway social situation where we humans expect to go one after another and not rush or cut the line. Both the traditional planner (red line) and the SAN planner (blue line) were given the same goal (represented as a red star) and start (START) locations. The traditional trajectory planner tried to steer the robot to the goal location and stopped at an inappropriate location (beside the first person in front of the door). However, the SAN planner steered the robot to an appropriate location, i.e., end of the line, positioning the robot behind the last person (social goal), considering personal space as well.

Figure 14(a) shows the behavior of our social planner and traditional planner in foining a group situation in simulation. Here, we considered an HRI situation where the robot is required to join a group of three people. However, this can be generalized to interact with more people. Both the traditional planner (red line) and the SAN planner (blue line) were given the same goal (represented as a red star) and start (START) locations. The trajectory planner steered the robot to position it at an awkward location (middle of an interacting group), as the traditional planner did not account for group proxemics and group dynamics. However, the SAN planner steered the robot to an appropriate location, i.e., a vacant spot in the circle, considering group proxemics.

Figure 14(b) shows a variation in the open spot where the robot needs to join. In this case, the open spot is in a tricky location, as the robot has to approach the group from the back. Our proposed approach found a way around the group to the social goal. However, the traditional planner leads the robot to the group's center while getting in between two people (blue and green shirts).

Figure 14(c) not only differs in the size of the circle formed but also is a variation of O-formation where the robot is leading the conversion opposing to joining a conversation. When joining a group for discussion, we tend to maintain a uniform spacing between every member of a group; whereas joining a group to lead the conversation, all the members of the group except the lead squish together so the leader can make eye contact with all the members (with as little field of view as possible). In this case, the traditional planner guides the robot to the center of the group, whereas the proposed method guides the robot to a social goal location where the robot can effectively interact with the group.

Figure 14(d) shows the results of our method implemented on a Pioneer robot executing an appropriate social behavior of joining a group. Both the traditional planner (red line) and the SAN planner (blue line) were given the same goal (represented as a red star) and start (START) locations. The trajectory planner steered the robot to position it at an inappropriate location (the middle of an interacting group), as the traditional planner did not account for group proxemics and group dynamics. However, the SAN planner steered the robot to an appropriate location, i.e., a vacant spot in the circle, considering group proxemics.

\subsection{Comparison Results}

We compare our optimization-based SAN approach with a recent inverse reinforcement learningbased SAN method [36]. Kollmitz et al. [36] investigated force control as a natural means to teach social trajectories to robots using inverse reinforcement learning trained using demonstrations. The IRL method learned hallway passing through demonstrated trajectories. We used the hallway passing scenario as a common task for all three planners, and the results for various metrics are 


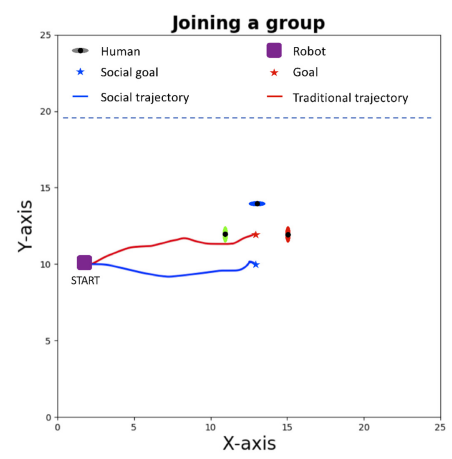

(a) Scenario 4: The robot is joining a group where the robot with SAN planner forms an O-formation in order to interact with the group. The traditional planner generates the red trajectory and places the robot in the center of the group. Proposed SAN planner generated the blue trajectory which leads the robot to form an O-formation.

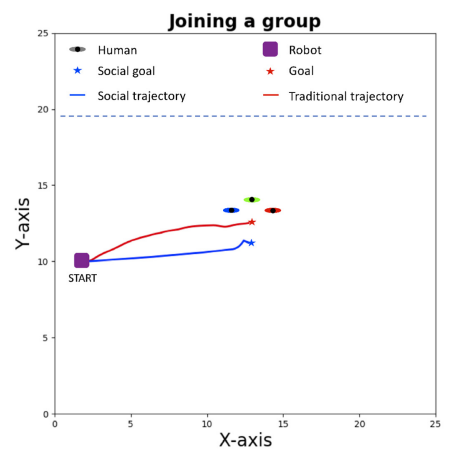

(c) Scenario 4 (robot leading the group's conversation): The traditional planner generated the red trajectory and placed the robot in the center of the group. Proposed approach generated the blue trajectory which leads the robot to form an O-formation (appropriate).

Fig. 14. Validation results of Scenario 4 (joining a group) in both simulation and real-world.

shown in Table 1 (average of five runs). The simulated task involves a robot passing a human in a hallway, the length of the hallway scenario (straight-line distance) is kept at five meters, and the robot meets a simulated human halfway through the hallway. We used the following metrics to compare our work:

(1) Total distance: Total distance traveled by the robot to reach the goal.

(2) Time to reach goal: Total time taken to reach the goal location.

(3) Lateral distance maintained: The lateral distance maintained by the robot when passing the human.

(4) No. of Proxemic intrusions: Number of times the robot intruded into the human's personal space. 
Table 1. Comparison of Traditional Navigation, IRL-based Social Navigation, and Our Approach on the Hallway Passing Scenario (Results Are Average of Five Runs)

\begin{tabular}{|cc|ccc|}
\hline Metric & Expected & Traditional & IRL & PaCcET \\
\hline \hline Total distance $\left(\boldsymbol{D}_{\boldsymbol{t}}\right)$ & LOW & $\mathbf{5 . 0 4 m}$ & $5.21 \mathrm{~m}$ & $5.34 \mathrm{~m}$ \\
\hline Time to reach goal $(\boldsymbol{t})$ & LOW & $\mathbf{1 3 . 2 s}$ & $15.94 \mathrm{~s}$ & $\mathbf{1 4 . 3 6}$ \\
\hline Lateral distance maintained $\left(\boldsymbol{D}_{\boldsymbol{l}}\right)$ & HIGH & $0.9 \mathrm{~m}$ & $1.01 \mathrm{~m}^{*}$ & $\mathbf{1 . 3 9 m}$ \\
\hline No. of Proxemic Intrusions $(\boldsymbol{N})$ & LOW & 5 & $\mathbf{0}^{*}$ & $\mathbf{0}$ \\
\hline Distance when robot deviated $\left(\boldsymbol{D}_{\boldsymbol{d}}\right)$ & HIGH & n/a & $1.8 \mathrm{~m}$ & $\mathbf{1 . 5 m}$ \\
\hline
\end{tabular}

*The number of proxemic intrusions into personal space is considered 0 for IRL-based method even though the IRL approach maintained an average lateral distance of $1.01 \mathrm{~m}$ only (the max range of personal space according to Hall's model of proxemics is 1.2 meters). This is because the participants trained the robot for their comfortable distance; hence, we assume the robot did not intrude the personal zone (Proxemic theory depends on many factors such as familiarity with robots).

(5) Distance when robot deviated: Distance from the human when the robots starts to deviate. This metric may contribute to the legibility and predictability of a robot trajectory [5, 19].

It is evident from Table 1 that the traditional planner outperformed other social navigation methods in terms of performance metrics such as distance traveled and time taken to reach the goal. However, traditional navigation methods under-performed in terms of social metrics such as lateral distance maintained, proxemic intrusions, and distance from a human when the robot started to show deviation in its path. However, it is worth noting that it is acceptable for a social robot to choose a performance-based sub-optimal path, as the quality of interaction takes precedence over performance. The distance traveled by the IRL-based SAN method is less than the distance traveled by our approach, and the difference is explained by the fact that our approach maintained a larger lateral distance when passing a person. On the time to reach goal metric, our method performed better, as our method is a modification of traditional navigation.

On social metrics, our method performed well on lateral distance maintained compared to the IRL and traditional navigation methods. Our experiments show that our approach and IRL-based approach reported zero proxemic intrusions into personal space (max range of 1.2 meters). However, it is worth noting that a person's comfortable personal space with a robot depends on numerous factors (both robot and human) such as gender, familiarity with robots [58]. The number of proxemic intrusions into personal space is considered zero for IRL-based method even though the IRL approach maintained an average lateral distance of $1.01 \mathrm{~m}$ only (the max range of personal space according to Hall's model of proxemics [28] is 1.2 meters). This is because the participants trained the robot for their comfortable distance; hence, we assume the robot did not intrude the personal zone. On the other social metric (distance from which the robot started to deviate), the IRL-based planner performed slightly better when compared to our approach, and we attribute this to the fact that the IRL-based social navigation is a global planner, whereas our approach to social navigation is at the local planning stage. Planning for social objectives with global planning happens at the beginning of the planning task, whereas planning for social objectives at a local planning stage happens as the robot is approaching a person. Hence, the IRL-based planner was able to deviate when passing the person much earlier than our approach. It is worth noting that both the social navigation methods outperformed the traditional planner, as the traditional planner did not even deviate from its planned trajectory, as it passed the human very closely. Such deviations before passing a person may lead to legibility and predictability of trajectories [19]. 


\section{DISCUSSION AND FUTURE WORK}

With a series of experiments in simulation and with a real robot using a multi-objective optimization tool like PaCcET at the local planning stage of navigation, we showed that social norms related to proxemics could be addressed in a SAR system, which in turn can aid the acceptance of robots in human environments. We showed that our approach could handle a simple, single-person interaction in a hallway scenario. We also showed that our approach could handle sub-scenarios (different types of interaction in a scenario) such as a stationary human in a hallway, passing behavior, and so on. Similarly, in an art-gallery situation, our methods showed that it is applicable for sub-scenarios such as presenting artwork and avoiding activity zones. We demonstrated the generalizability of our approach by introducing multiple humans in complex social scenarios with multiple features such as interpersonal distances, group proxemics, activity zone, and social goal distance (adherence to social goal). Finally, we showed that our modified local planner could adjust to changes in scenarios such as the location of people, different line formations, and O-formations.

This work dealt with low-level decisions of which future trajectory points are better for the given interaction scenario. One assumption in this work is that the robot has prior knowledge of the ongoing interaction. We will extend this work using a knowledge graph approach [55], implementing a high-level decision-making system that can select the crucial objectives for the quality of HRI for an autonomously sensed scenario [5]. We will extend this work by validating this system using its conformity to social metrics defined by the social parameters we discussed and surveying the perception of social intelligence of the resultant behavior. We plan to utilize not just the distance-based feature but also features related to orientation, such as heading angle, heading difference between the robot and the people/group, and so on, along with features related to the environment, such as the position of the agents (robot and people) in the environment (for example, in a hallway, distance from the right side of the hallway) [54].

When examining the social impact of a SAN system, it is important that any instruments used are properly assessing social intelligence. Kruse et al. [39] identified Comfort, Sociability, and Naturalness as challenges that SAN planners should tackle in a collaborative human environment [39]. We identified other challenges such as predictability, legibility, safety, acceptance, and so on, and working on providing clear definitions and metrics/methods for measuring them. Perceived Social Intelligence (PSI) is an important parameter we identified, which has importance in robot motion in human environments. Social intelligence is the ability to interact effectively with others to accomplish one's goals [24]. Social intelligence is critically important for any robot that will be around people, whether engaged in social or non-social tasks. Some aspects of robotic social intelligence have been included in HRI research [10, 32, 45, 47], but current measures are brief and often include extraneous variables. We designed a comprehensive instrument for measuring the PSI of robots [7-9], which should more precisely measure the social impact of our approach on people in the robot's environment and people observing those interactions $[6,33]$.

\section{CONCLUSION}

We presented a novel approach to the socially aware navigation (SAN) problem at a local planning stage by transforming the Pareto front to an objective space (forced to be convex) using Pareto Concavity Elimination Transformation (PaCcET) method. PaCcET was implemented in local planning of well-established ROS navigation stack to deal with spatial communication at a low-level planning stage. We validated the developed system both in simulation and on a mobile robot to show the applicability of our proposed approach with multiple scenarios involving multiple humans. A follow-up study will investigate the social aspect of the navigation behaviors using scales that already exist and new scales that our group is currently investigating. 


\section{ACKNOWLEDGMENTS}

We would like to acknowledge the help of Vineeth Rajamohan, Fausto Vega, Ashish Kasar, Athira Pillai, and Andrew Palmer.

\section{REFERENCES}

[1] Alexandre Alahi, Kratarth Goel, Vignesh Ramanathan, Alexandre Robicquet, Li Fei-Fei, and Silvio Savarese. 2016. Social LSTM: Human trajectory prediction in crowded spaces. In Proceedings of the IEEE Conference on Computer Vision and Pattern Recognition. IEEE, 961-971.

[2] Javier Alonso-Mora, Paul Beardsley, and Roland Siegwart. 2018. Cooperative collision avoidance for nonholonomic robots. IEEE Trans. Robot.ics 34, 2 (2018), 404-420.

[3] Anoop Aroor, Susan L. Epstein, and Raj Korpan. 2018. Online learning for crowd-sensitive path planning. In Proceedings of the 17th International Conference on Autonomous Agents and MultiAgent Systems. International Foundation for Autonomous Agents and Multiagent Systems, ACM, New York, NY, 1702-1710.

[4] Richard J. Balling, John T. Taber, Michael R. Brown, and Kirsten Day. 1999. Multiobjective urban planning using genetic algorithm. F. Urb. Plan. Dev. 125, 2 (1999), 86-99.

[5] Santosh Balajee Banisetty and David Feil-Seifer. 2018. Towards a unified planner for socially-aware navigation. In Proceedings of the AAAI Fall Symposium Series (AI-HRI'18).

[6] Santosh Balajee Banisetty and Tom Williams. 2021. Implicit communication through social distancing: Can social navigation communicate social norms? In Proceedings of the ACM/IEEE International Conference on Human-robot Interaction (HRI LBRs'21).

[7] Kimberly Barchard, Leiszle Lapping-Carr, Shane Westfall, and David Feil-Seifer. 2019. Perceived social intelligence of robots. In Society for Personality and Social Psychology. Society for Personality and Social Psychology, Portland, Oregon. https://spsp.org/.

[8] Kimberly A. Barchard, Leiszle Lapping-Carr, R. Shane Westfall, Santosh Balajee Banisetty, and David Feil-Seifer. 2018 Perceived Social Intelligence (PSI) Scales test manual. Retrieved from https://ipip.ori.org/newMultipleconstructs.htm.

[9] Kimberly A. Barchard, Leiszle Lapping-Carr, R. Shane Westfall, Andrea Fink-Armold, Santosh Balajee Banisetty, and David Feil-Seifer. 2020. Measuring the perceived social intelligence of robots. ACM Trans. Hum.-robot Interact. 9, 4 (2020), 1-29.

[10] Christoph Bartneck, Dana Kulić, Elizabeth Croft, and Susana Zoghbi. 2009. Measurement instruments for the anthropomorphism, animacy, likeability, perceived intelligence, and perceived safety of robots. Int. f. Soc. Robot. 1, 1 (2009), 71-81.

[11] Wolfram Burgard, Armin B. Cremers, Dieter Fox, Dirk Hähnel, Gerhard Lakemeyer, Dirk Schulz, Walter Steiner, and Sebastian Thrun. 1998. The interactive museum tour-guide robot. In Proceedings of the Conference on Innovative Applications of Artificial Intelligence. ACM, 11-18.

[12] Wolfram Burgard, Armin B. Cremers, Dieter Fox, Dirk Hähnel, Gerhard Lakemeyer, Dirk Schulz, Walter Steiner, and Sebastian Thrun. 1999. Experiences with an interactive museum tour-guide robot. Artif. Intell. 114, 1 (1999), 3-55.

[13] Zachary Carlson, Louise Lemmon, MacCallister Higgins, David Frank, Roya Salek Shahrezaie, and David Feil-Seifer. 2019. Perceived mistreatment and emotional capability following aggressive treatment of robots and computers. Int f. Soc. Robot. (24 Oct. 2019). DOI : https://doi.org/10.1007/s12369-019-00599-8

[14] C. A. Coello and Alan D. Christiansen. 2000. Multiobjective optimization of trusses using genetic algorithms. Comput. Struct. 75, 6 (2000), 647-660.

[15] Carlos A. Coello. 1999. A comprehensive survey of evolutionary-based multiobjective optimization techniques. Knowl. Inf. Syst. 1, 3 (1999), 269-308.

[16] Mitchell Colby, Logan Yliniemi, Paolo Pezzini, David Tucker, Kenneth Mark Bryden, and Kagan Tumer. 2016. Multiobjective neuroevolutionary control for a fuel cell turbine hybrid energy system. In Proceedings of the Genetic and Evolutionary Computation Conference. ACM, 877-884.

[17] Christian Dondrup and Marc Hanheide. 2016. Qualitative constraints for human-aware robot navigation using velocity costmaps. In Proceedings of the 25th IEEE International Symposium on Robot and Human Interactive Communication (RO-MAN'16). IEEE, New York, NY, 586-592.

[18] Brendan Dorsey. 2018. Smart Luggage Robot. Retrieved from https://thepointsguy.com/2018/01/autonmous-smartluggage-premiere-ces/.

[19] Anca D. Dragan, Kenton C. T. Lee, and Siddhartha S. Srinivasa. 2013. Legibility and predictability of robot motion. In Proceedings of the 8th ACM/IEEE International Conference on Human-robot Interaction (HRI'13). IEEE, 301-308.

[20] David Feil-Seifer and Maja Matarić. 2012. Distance-based computational models for facilitating robot interaction with children. F. Hum.-robot Interact. 1, 1 (July 2012), 55-77. DOI : https://doi.org/10.5898/JHRI.1.1.Feil-Seifer

[21] David Feil-Seifer and Maja J. Mataric. 2005. Defining socially assistive robotics. In Proceedings of the 9th International Conference on Rehabilitation Robotics. IEEE, 465-468.

ACM Transactions on Interactive Intelligent Systems, Vol. 11, No. 2, Article 15. Publication date: June 2021. 
[22] Gonzalo Ferrer, Anais Garrell, and Alberto Sanfeliu. 2013. Robot companion: A social-force based approach with human awareness-navigation in crowded environments. In Proceedings of the IEEE/RSf International Conference on Intelligent Robots and Systems (IROS'13). IEEE, 1688-1694.

[23] Gonzalo Ferrer, Anaís Garrell Zulueta, Fernando Herrero Cotarelo, and Alberto Sanfeliu. 2017. Robot social-aware navigation framework to accompany people walking side-by-side. Auton. Robots 41, 4 (2017), 775-793.

[24] Martin E. Ford and Marie S. Tisak. 1983. A further search for social intelligence. f. Educ. Psychol. 75, 2 (1983), 196.

[25] Scott Forer, Santosh Balajee Banisetty, Logan Yliniemi, Monica Nicolescu, and David Feil-Seifer. 2018. Socially-aware navigation using non-linear multi-objective optimization. In Proceedings of the IEEE/RSf International Conference on Intelligent Robots and Systems (IROS'18). IEEE, 1-9.

[26] Brian Gerkey, Richard T. Vaughan, and Andrew Howard. 2003. The player/stage project: Tools for multi-robot and distributed sensor systems. In Proceedings of the 11th International Conference on Advanced Robotics, Vol. 1. IEEE, 317-323.

[27] Javier V. Gómez, Nikolaos Mavridis, and Santiago Garrido. 2014. Fast marching solution for the social path planning problem. In Proceedings of the IEEE International Conference on Robotics and Automation (ICRA'14). IEEE, 1871-1876.

[28] Edward Twitchell Hall. 1966. The Hidden Dimension. Doubleday \& Co, Garden City, NY.

[29] Mahmoud Hamandi, Mike D’Arcy, and Pooyan Fazli. 2018. DeepMoTIon: Learning to Navigate Like Humans. arxiv:cs.RO/1803.03719 (2018).

[30] Isobel Asher Hamilton. 2018. People kicking these food delivery robots is an early insight into how cruel humans could be to robots. Retrieved from https://www.businessinsider.com/people-are-kicking-starship-technologiesfood-delivery-robots-2018-6? $\mathrm{r}=\mathrm{US} \& \mathrm{IR}=\mathrm{T}$.

[31] Dirk Helbing and Peter Molnar. 1995. Social force model for pedestrian dynamics. Phys. Rev. E 51, 5 (1995), 4282.

[32] Chin-Chang Ho and Karl F. MacDorman. 2010. Revisiting the uncanny valley theory: Developing and validating an alternative to the Godspeed indices. Comput. Hum. Behav. 26, 6 (2010), 1508-1518.

[33] AnaLisa Honour, Santosh Balajee Banisetty, and David Feil-Seifer. 2021. Perceived social intelligence as evaluation of socially navigation. In Proceedings of the ACM/IEEE International Conference on Human-robot Interaction (HRI LBRs'21).

[34] Beomjoon Kim and Joelle Pineau. 2016. Socially adaptive path planning in human environments using inverse reinforcement learning. Int. F. Soc. Robot. 8, 1 (2016), 51-66.

[35] Hasan Kıvrak and Hatice Köse. 2018. Social robot navigation in human-robot interactive environments: Social force model approach. In Proceedings of the 26th Signal Processing and Communications Applications Conference (SIU'16). IEEE, $1-4$.

[36] Marina Kollmitz, Torsten Koller, Joschka Boedecker, and Wolfram Burgard. 2020. Learning human-aware robot navigation from physical interaction via inverse reinforcement learning. In Proceedings of the IEEE/RSF International Conference on Intelligent Robots and Systems (IROS’20). Retrieved from http://ais.informatik.uni-freiburg.de/publications/ papers/kollmitz20iros.pdf.

[37] Henrik Kretzschmar, Markus Spies, Christoph Sprunk, and Wolfram Burgard. 2016. Socially compliant mobile robot navigation via inverse reinforcement learning. Int. f. Soc. Robot. 35, 11 (2016), 1289-1307.

[38] Thibault Kruse, Alexandra Kirsch, Harmish Khambhaita, and Rachid Alami. 2014. Evaluating directional cost models in navigation. In Proceedings of the ACM/IEEE International Conference on Human-robot Interaction. ACM, 350-357.

[39] Thibault Kruse, Amit Kumar Pandey, Rachid Alami, and Alexandra Kirsch. 2013. Human-aware robot navigation: A survey. Robot. Auton. Syst. 61, 12 (2013), 1726-1743.

[40] Angus Leigh, Joelle Pineau, Nicolas Olmedo, and Hong Zhang. 2015. Person tracking and following with 2D laser scanners. In Proceedings of the IEEE International Conference on Robotics and Automation (ICRA'15). IEEE, 726-733.

[41] Felix Lindner and Carola Eschenbach. 2011. Towards a formalization of social spaces for socially aware robots. In Spatial Information Theory, Max Egenhofer, Nicholas Giudice, Reinhard Moratz, and Michael Worboys (Eds.). Springer Berlin, 283-303.

[42] Eitan Marder-Eppstein, Eric Berger, Tully Foote, Brian Gerkey, and Kurt Konolige. 2010. The office marathon: Robust navigation in an indoor office environment. In Proceedings of the IEEE International Conference on Robotics and Automation (ICRA'10). IEEE, 300-307.

[43] R. Timothy Marler and Jasbir S. Arora. 2004. Survey of multi-objective optimization methods for engineering. Struct. Multidisc. Optim. 26, 6 (2004), 369-395.

[44] Achille Messac and Philip D. Hattis. 1996. Physical programming design optimization for high speed civil transport. 7. Aircraft 33, 2 (1996), 446-449.

[45] Lilia Moshkina. 2012. Reusable semantic differential scales for measuring social response to robots. In Proceedings of the Workshop on Performance Metrics for Intelligent Systems. ACM, 89-94.

[46] Bilge Mutlu and Jodi Forlizzi. 2008. Robots in organizations: The role of workflow, social, and environmental factors in human-robot interaction. In Proceedings of the International Conference on Human-robot Interaction (HRI'08). ACM, 287-294. 
[47] Tatsuya Nomura, Tomohiro Suzuki, Takayuki Kanda, and Kensuke Kato. 2006. Measurement of negative attitudes toward robots. Interact. Stud. 7, 3 (2006), 437-454.

[48] Billy Okal and Kai O. Arras. 2016. Learning socially normative robot navigation behaviors with Bayesian inverse reinforcement learning. In Proceedings of the IEEE International Conference on Robotics and Automation (ICRA'16) IEEE, 2889-2895.

[49] Vineeth Rajamohan, Connor Scully-Allison, Sergiu Dascalu, and David Feil-Seifer. 2019. Factors influencing the human preferred interaction distance. In Proceedings of the IEEE International Conference on Robot and Human Interactive Communication (RO-MAN'19). IEEE.

[50] Jorge Rios-Martinez, Anne Spalanzani, and Christian Laugier. 2011. Understanding human interaction for probabilistic autonomous navigation using Risk-RRT approach. In Proceedings of the IEEE/RSF International Conference on Intelligent Robots and Systems. IEEE, 2014-2019.

[51] Jorge Rios-Martinez, Anne Spalanzani, and Christian Laugier. 2015. From proxemics theory to socially-aware navigation: A survey. Int. J. Soc. Robot. 7, 2 (2015), 137-153

[52] V. Sarfi and H. Livani. 2017. A novel multi-objective security-constrained power management for isolated microgrids in all-electric ships. In Proceedings of the IEEE Electric Ship Technologies Symposium (ESTS'17). IEEE, 148-155. DOI : https://doi.org/10.1109/ESTS.2017.8069273

[53] Vahid Sarfi, Hanif Livani, and Logan Yliniemi. 2017. A novel multi-objective security-constrained power management for isolated microgrids in all-electric ships. In Proceedings of the IEEE Electric Ship Technologies Symposium (ESTS'17). IEEE, Arlington, VA, 148-155.

[54] Meera Sebastian, Santosh Balajee Banisetty, and David Feil-Seifer. 2017. Socially-aware navigation planner using models of human-human interaction. In Proceedings of the International Symposium on Robot and Human Interactive Communication (RO-MAN'17). IEEE, 405-410. DOI : https://doi.org/10.1109/ROMAN.2017.8172334

[55] Roya Salek Shahrezaie, Santosh Balajee Banisetty, and David Feil-Seifer. 2021. Towards deep reasoning on social rules for socially aware navigation. In Proceedings of the ACM/IEEE International Conference on Human-robot Interaction (HRI LBRs'21).

[56] Grimaldo Silva and Thierry Fraichard. 2017. Human robot motion: A shared effort approach. In Proceedings of the European Conference on Mobile Robots (ECMR'17). IEEE, 1-6.

[57] Stefan-Daniel Suvei, Jered Vroon, Vella V. Somoza Sanchéz, Leon Bodenhagen, Gwenn Englebienne, Norbert Krüger, and Vanessa Evers. 2018. "I would like to get close to you": Making robot personal space invasion less intrusive with a social gaze cue. In Proceedings of the International Conference on Universal Access in Human-Computer Interaction. Virtual, Augmented, and Intelligent Environments, Margherita Antona and Constantine Stephanidis (Eds.). Springer International Publishing, Cham, 366-385.

[58] Leila Takayama and Caroline Pantofaru. 2009. Influences on proxemic behaviors in human-robot interaction. In Proceedings of the IEEE/RSf International Conference on Intelligent Robots and Systems. IEEE, 5495-5502.

[59] Zheng-Hua Tan, Nicolai Bæk Thomsen, Xiaodong Duan, Evgenios Vlachos, Sven Ewan Shepstone, Morten Højfeldt Rasmussen, and Jesper Lisby Højvang. 2018. iSocioBot: A multimodal interactive social robot. Int. F. Soc. Robot. 10, 1 (2018), 5-19.

[60] S. Thrun, M. Bennewitz, W. Burgard, A. B. Cremers, F. Dellaert, D. Fox, D. Hahnel, C. Rosenberg, N. Roy, J. Schulte, and D. Schulz. 1999. MINERVA: A second-generation museum tour-guide robot. In Proceedings of the IEEE International Conference on Robotics and Automation, Vol. 3. IEEE, 1999-2005. DOI : https://doi.org/10.1109/ROBOT.1999.770401

[61] Xuan-Tung Truong and Trung-Dung Ngo. 2016. Dynamic social zone based mobile robot navigation for human comfortable safety in social environments. Int. F. Soc. Robot. 8, 5 (2016), 663-684.

[62] Annemarie Turnwald and Dirk Wollherr. 2019. Human-like motion planning based on game theoretic decision making. Int. F. Soc. Robot. 11, 1 (01 Jan. 2019), 151-170. DOI : https://doi.org/10.1007/s12369-018-0487-2

[63] Logan Yliniemi. 2014. Considerations for multiagent multi-objective systems. In Proceedings of the International Conference on Autonomous Agents and Multi-agent Systems. International Foundation for Autonomous Agents and Multiagent Systems, ACM, 1719-1720.

[64] Logan Yliniemi and Kagan Tumer. 2014. PaCcET: An objective space transformation to iteratively convexify the Pareto front. In Simulated Evolution and Learning, Grant Dick, Will N. Browne, Peter Whigham, Mengjie Zhang, Lam Thu Bui, Hisao Ishibuchi, Yaochu Jin, Xiaodong Li, Yuhui Shi, Pramod Singh, Kay Chen Tan, and Ke Tang (Eds.). Springer International Publishing, Cham, 204-215.

[65] L. Yliniemi and K. Tumer. 2015. Complete coverage in the multi-objective PaCcET framework. In Proceedings of the Genetic and Evolutionary Computation Conference. ACM, 1525-1526.

Received April 2019; revised December 2020; accepted February 2021 